\title{
Erratum: Scattering lengths for two pseudoscalar meson systems [Phys. Rev. D 89, 054502 (2014)]
}

Kiyoshi Sasaki, Naruhito Ishizuka, Makoto Oka, and Takeshi Yamazaki

(PACS-CS Collaboration)

(Received 15 December 2021; published 10 January 2022)

DOI: 10.1103/PhysRevD.105.019901

The last term in the square bracket of Eq. (A9) was given by $10 m_{K}^{2} / 9$. However, the correct expression is $7 m_{K}^{2} / 9$. Along with this correction, we have redone the chiral analysis. The LECs and the scattering lengths at the physical point are shifted at most by one standard error, but we stress that the main argument is not changed.

The final results for the scattering lengths at the physical point are summarized as

$$
\begin{aligned}
a_{0}^{(2)} m_{\pi} & =-0.04243(22)(43) \\
a_{0}^{(1)} m_{K} & =-0.312(17)(31) \\
a_{0}^{(3 / 2)} \mu_{\pi K} & =-0.0477(27)(20) \\
a_{0}^{(1 / 2)} \mu_{\pi K} & =0.150(16)(37),
\end{aligned}
$$

and

$$
\begin{aligned}
& a_{0}^{(3 / 2)} m_{\pi}=-0.0612(35)(26) \\
& a_{0}^{(1 / 2)} m_{\pi}=0.193(21)(47),
\end{aligned}
$$

corresponding to Eqs. (53) and (63), respectively. The values of item "The present work" in Table XI must be revised by these values.

The systematic error from the uncertainty of $f_{\pi}$ and $f_{K}$ is almost the same size as the previous one. On the other hand, we need attention for the systematic error from the higher order effects of ChPT. For the $\pi K(I=1 / 2)$ system, the shift of the $\mathcal{O}\left(p^{4}\right)$ value causes an increase in it. The counterpart of Eq. (62) becomes

$$
\begin{aligned}
a_{0}^{(2)} m_{\pi} & =-0.04243(22) \pm 0.00029 \\
a_{0}^{(1)} m_{K} & =-0.312(17) \pm 0.020 \\
a_{0}^{(3 / 2)} \mu_{\pi K} & =-0.0477(27) \pm 0.0001 \\
a_{0}^{(1 / 2)} \mu_{\pi K} & =0.150(16) \pm 0.035 .
\end{aligned}
$$

Figures 9-15 are affected by this correction, but the effect is small. We will only show the revised versions of Figs. 11, 12, and 15 because they illustrate the most important results for the chiral analysis. Tables IX and X are also slightly affected. However, we will only show the revised version of Table IX for the same reason. 


\section{Data Set A}
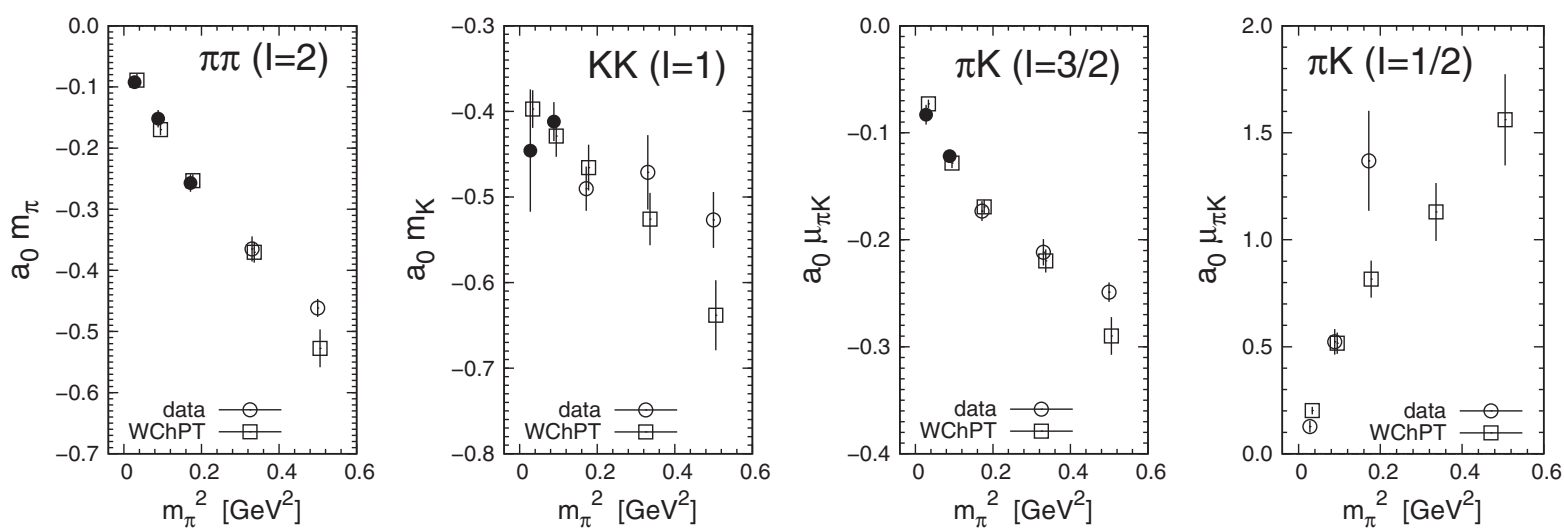

\section{Data Set B}
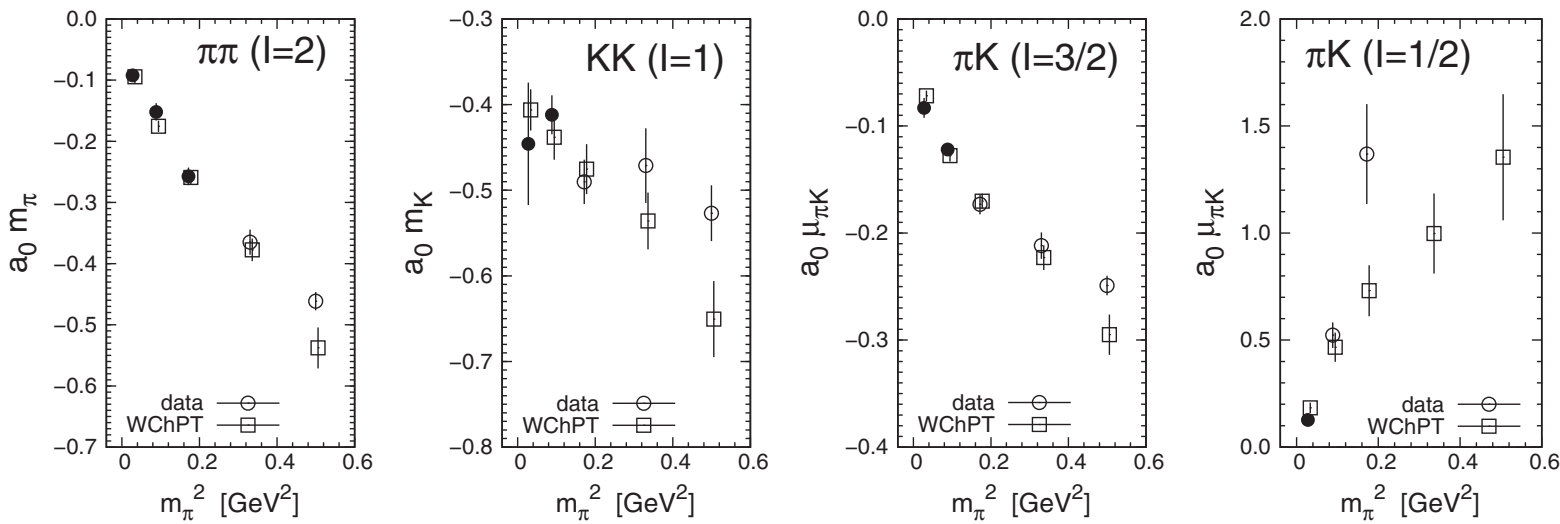

\section{Data Set C}
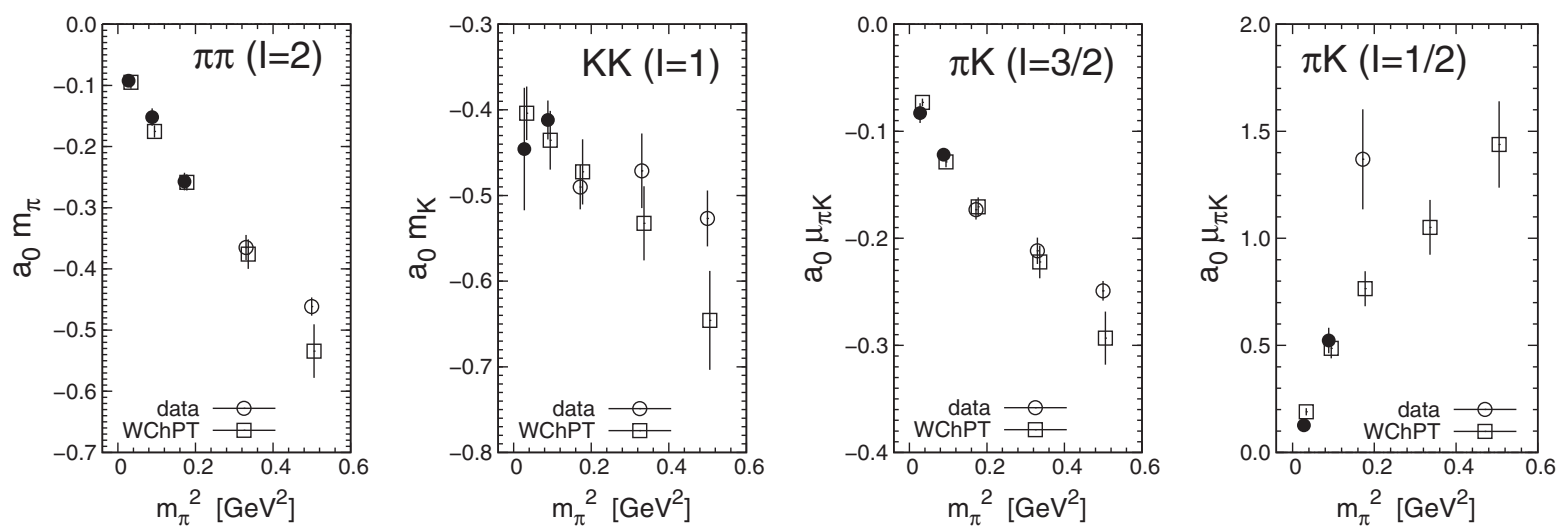

FIG. 11. Fitting results of the $\mathcal{O}\left(p^{4}\right)$ WChPT fit with the data sets A, B, and C. The data are represented by circles, and those used in the fit especially, are denoted by filled symbols. The fitting results by the WChPT formulas are denoted by squares.

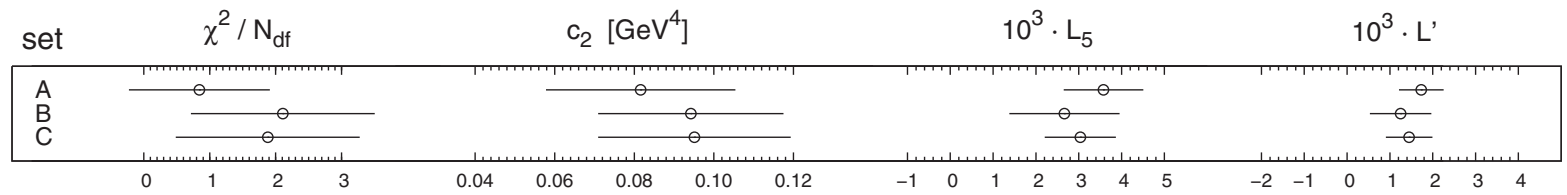

FIG. 12. $\chi^{2} / N_{\text {d.o.f }}$ and LECs $\left(c_{2}, 10^{3} \cdot L_{5}\right.$, and $\left.10^{3} \cdot L^{\prime}\right)$ determined in the $\mathcal{O}\left(p^{4}\right)$ WChPT fit with the data sets A, B, and C. 


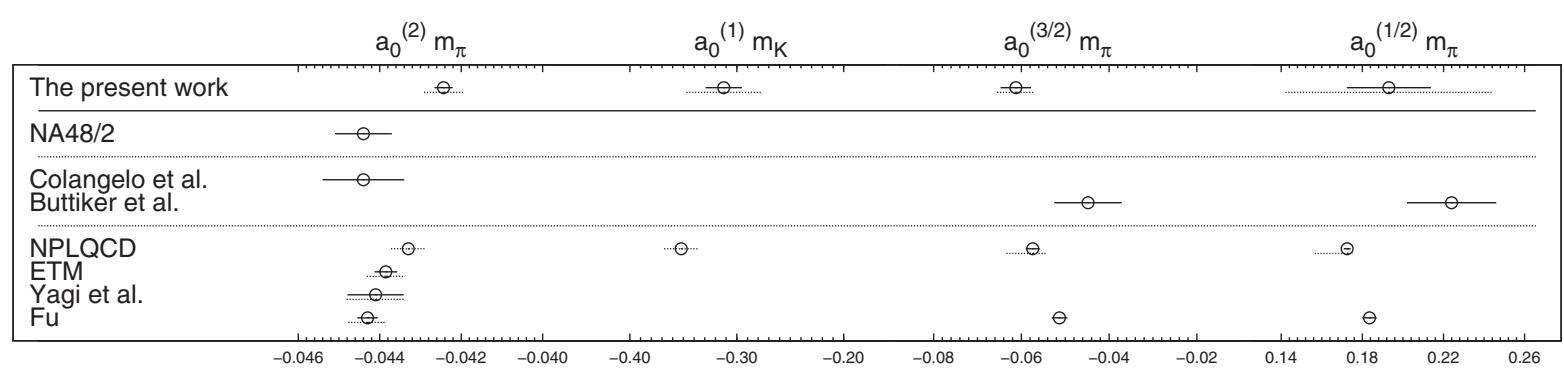

FIG. 15. $a_{0}^{(2)} m_{\pi}, a_{0}^{(1)} m_{K}, a_{0}^{(3 / 2)} m_{\pi}$, and $a_{0}^{(1 / 2)} m_{\pi}$ in the present work are shown in a comparison with the previous works. As the previous works, we refer the experimental value by NA48/2 [49], the phenomenological evaluations by Colangelo et al. [50] and Büttiker et al. [51], and the lattice calculations by the NPLQCD Collaboration [12, 15, 18], the ETM Collaboration [13], Yagi et al. [14], and $\mathrm{Fu}[5,19]$.

TABLE IX. $\quad \chi^{2} / N_{\text {d.o.f }}$ and LECs obtained from the $\mathcal{O}\left(p^{4}\right) S U(3)$ WChPT fits for data sets A, B, and C. The scattering lengths at the physical point are also shown.

\begin{tabular}{lccc}
\hline \hline Data set & $\mathrm{A}$ & $\mathrm{B}$ & $\mathrm{C}$ \\
\hline$\chi^{2} / N_{\mathrm{df}}$ & $0.8(1.1)$ & $2.1(1.4)$ & $1.9(1.4)$ \\
$c_{2}\left[\mathrm{GeV}^{4}\right]$ & $0.082(24)$ & $0.094(23)$ & $0.095(24)$ \\
$10^{3} \cdot L_{5}$ & $3.58(93)$ & $2.7(1.3)$ & $3.04(83)$ \\
$10^{3} \cdot L^{\prime}$ & $1.73(52)$ & $1.25(72)$ & $1.45(54)$ \\
\hline$a_{0}^{(2)} m_{\pi}$ & $-0.04239(21)$ & $-0.04243(22)$ & $-0.04241(29)$ \\
\hline$a_{0}^{(1)} m_{K}$ & $-0.309(16)$ & $-0.312(17)$ & $-0.343(23)$ \\
$a_{0}^{(1)} m_{\pi}$ & $-0.0874(45)$ & $-0.0885(48)$ & $-0.0972(66)$ \\
\hline$a_{0}^{(3 / 2)} \mu_{\pi K}$ & $-0.0497(20)$ & $-0.0477(27)$ & $-0.0485(15)$ \\
$a_{0}^{(3 / 2)} m_{\pi}$ & $-0.0638(26)$ & $-0.0612(35)$ & $-0.0623(19)$ \\
\hline$a_{0}^{(1 / 2)} \mu_{\pi K}$ & $0.162(12)$ & $0.150(16)$ & $0.155(11)$ \\
$a_{0}^{(1 / 2)} m_{\pi}$ & $0.208(15)$ & $0.193(21)$ & $0.199(14)$ \\
\hline \hline
\end{tabular}

\section{ACKNOWLEDGMENTS}

We would like to thank A. Walker-Loud for pointing out the mistake in Eq. (A9) and having a fruitful discussion. 